\title{
Strategies to optimize a pediatric magnetic resonance imaging service
}

\author{
$\operatorname{Limin}_{\mathrm{Xu}}{ }^{1} \cdot$ Jeremy Herrington ${ }^{1} \cdot$ Kellie Cahill $^{1} \cdot$ Seretha Risacher ${ }^{1} \cdot$ Michael S. Gee $^{1} \mathbb{D}$
}

Received: 9 November 2020 / Revised: 29 January 2021 / Accepted: 16 March 2021 / Published online: 15 April 2021

(C) The Author(s), under exclusive licence to Springer-Verlag GmbH Germany, part of Springer Nature 2021

\begin{abstract}
A pediatric MRI service is a vital component of a successful radiology department. Building an efficient and effective pediatric MRI service is a multifaceted process that requires detailed planning for considerations related to finance, operations, quality and safety, and process improvement. These are compounded by the unique challenges of caring for pediatric patients, particularly in the setting of the recent coronavirus disease 2019 (COVID-19) pandemic. In addition to material resources, a successful pediatric MRI service depends on a collaborative team consisting of radiologists, physicists, technologists, nurses and vendor specialists, among others, to identify and resolve challenges and to strive for continued improvement. This article provides an overview of the factors involved in both starting and optimizing a pediatric MRI service, including commonly encountered obstacles and some proposed solutions to address them.
\end{abstract}

Keywords Body $\cdot$ Children $\cdot$ Magnetic resonance imaging $\cdot$ Operations $\cdot$ Pediatric radiology $\cdot$ Service

\section{Introduction}

Developing a successful pediatric MRI service is a complex, multifaceted process that involves financial considerations for equipment purchases, service contracts and upgrades; operational considerations regarding quality, safety, workflow and interpretation; as well as a plan for assessing key performance indicators and improving processes. Perhaps most important, a dedicated MR team with expertise in image acquisition, operational logistics and safety is essential to recognizing opportunities for continued improvement in patient care delivery. Furthermore, distinctive challenges of caring for pediatric patients require additional consideration, for example the need for examination under sedation in a subset of children. These obstacles are compounded by the recent coronavirus disease 2019 (COVID-19) pandemic.

Michael S. Gee

msgee@mgh.harvard.edu

1 Department of Radiology, Massachusetts General Hospital, Harvard Medical School, 55 Fruit St., Ellison 237, Boston, MA 02114, USA

\section{Financial considerations when building a magnetic resonance imaging service}

Adequate financial support is crucial to the success of an MRI service. Initial costs related to setup of a new scanner include siting of the physical location, purchase of scanner equipment, and construction [1]. Once a scanner is set up, ongoing costs include salary support for MRI staff including technologists, physicists and radiologists; service and maintenance expenses; and any costs associated with physical space and equipment leasing, as well as periodic upgrades of software and coils. Because of the high costs associated with new MR scanners, equipment purchases might not fit into the annual radiology department operating budget and might require a separate capital planning process [2]. New MR equipment can be leased rather than purchased, which offers the advantages of lower annual expenditures and the opportunity to upgrade older scanners in a timelier fashion compared with traditional equipment purchasing. Because of the high level of technological innovation in pediatric MRI [3], MR equipment should be upgraded every few years, so financial planning for an MRI service should include planning for the immediate (1year), medium (5-year) and long-term (10-year) horizons. 
To provide an objective value measure of existing MR equipment and prioritize scanners for upgrade or replacement, an equipment useful-life process should be developed to aid in capital planning that includes grading of individual pieces of equipment on a regular basis [4]. Such a grading system should include equipment age and estimation of remaining useful life, qualitative factors such as image quality and safety ratings, and quantitative factors such as utilization rate, scan time and technical specifications [2]. One such list of MRI hardware and technical specifications was created by the Royal College of Radiologists for MRI equipment planning for the United Kingdom National Health Service [5]. A grade or score is issued for each existing scanner in an MR inventory to help identify areas of need and objectively track scanner performance longitudinally. Based on grade trends, specific features can be targeted for upgrade and a timeline for upgrade or replacement can be derived, which further aids in prioritizing current and future capital planning.

In terms of MR upgrades, scanner replacement is the most expensive option ( $>$ \$1 million USD) but provides the largest improvement in scanner capabilities. Multiple MR vendors offer limited upgrade options that keep the existing magnet in place while replacing the surrounding equipment including electronics, gradients and workstation (e.g., Fit, Siemens, Malvern, PA; Lift, GE Healthcare, Waukesha, WI). This option offers reductions in cost and construction downtime compared with a full scanner replacement. Less costly upgrade options $(\$ 10,000-\$ 100,000$ USD) include software, application and gradient coil replacements that can improve scanning time, image quality and data transmission efficiency. Many additional factors must be considered when replacing existing magnets, as well, that influence capital planning. In some cases, magnetic shielding also requires concurrent updating because these generally deteriorate over 1520 years, and power sources might need to be modified to provide sufficient voltage to support the upgraded equipment. Finally, physical requirements for adequate swing space and decanting volume during construction need to be factored, and this can also influence the decision to upgrade existing equipment or install new MR scanners.

Magnetic resonance vendor relationships can further influence the cost and choice of equipment installation and upgrades. There are advantages and disadvantages to single or multiple vendor relationships (Table 1). For example, working with a single vendor can streamline technologist training and equipment service, as well as provide long-term savings with a bundled service contract. However, a competitive market allows a multi-vendor model to provide competitive purchase prices and scanner-specific customization. Of course, it is acknowledged that choice of vendor for a clinical MRI service might be driven primarily by financial and other institution-specific factors (e.g., a common vendor for MR and CT or US), so this relationship is likely to be fixed for a given radiology department.

\section{Quality and safety considerations}

A robust quality management program consisting of quality control, quality assurance and quality improvement is crucial to ensure diagnostic image quality of a pediatric MRI service $[6,7]$. Quality control ensures that study images are of diagnostic quality; quality assurance involves ongoing review to ensure quality standards are consistently met; and quality improvement is a data-driven process by which process improvement strategies are identified and implemented. Discussion of image quality should also extend to equipment outside the MR scanner itself to include the required volume of surface coils and other ancillary equipment. It is important to note that having the best MRI equipment does not guarantee improvement in an MRI clinical service. For example, mean scan times for a given MR scanner are influenced greatly by patient type and study complexity, which can far outweigh equipment-specific factors. Scanners that accommodate a mixed patient population, including inpatients and sedation cases, will naturally have slower throughput and room turnover compared with scanners in outpatient locations. In addition, certain body parts (e.g., brain, knee) have shorter protocols compared with others (e.g., abdomen, whole body) and might require less real-time radiologist monitoring, implying that the referral base and exam mix of a given scanner will impact clinical volume and scheduled timeslot duration.

An active and engaged MRI safety committee is central to assuring safe operation of an MRI service. Routine quality and safety meetings are needed, during which the MRI safety committee reviews safety reports and other feedback to identify causes of error and opportunities for quality improvement [8]. Addressing safety considerations also requires advanced training in MRI safety for non-radiology care team members who accompany the patient during the study, restricted access to MRI safety zones for non-clinical personnel such as security and facility maintenance staff, and a rigorous process for screening for external and internal metal implants and metallic foreign bodies. Additionally, a protocol for performing MRI in children with implanted devices such as nerve stimulators and cardiac electronic devices must also be established, often in conjunction with the cardiology or electrophysiology service or device vendors [9]. Improper preparation or nonadherence to safety protocols in the patient preparation phase can lead to unplanned down time and potential for study cancellation.

Increased utilization of general anesthesia is a unique concern in pediatric MRI, especially in children younger than 6 years. Therefore, there are additional considerations unique to establishing a pediatric anesthesia MRI service from an operational perspective. These considerations include ensuring adequate staffing and scheduling of nurses and anesthetists, which is often a collaborative effort between radiology and anesthesiology departments [10]. Because of the fasting 
Table 1 Advantages of singleversus multi-vendor MRI service approach

\begin{tabular}{ll}
\hline Single-vendor & Multi-vendor \\
\hline $\begin{array}{c}\text { - Longitudinal relationship: coordination of } \\
\text { clinical and research efforts }\end{array}$ & $\begin{array}{c}\text { - Leveraging strengths of each vendor (compressed sensing, } \\
\text { deep learning/artificial intelligence, multi-contrast sequences, } \\
\text { automated workflow) } \\
\text { More vendor contacts: early access to latest } \\
\text { products and works in progress }\end{array}$ \\
$\begin{array}{c}\text { - Dedicated vendor resources (e.g., onsite } \\
\text { physicist and engineer) }\end{array}$ & $\begin{array}{l}\text { - Scanner-specific protocols and works in progress sequences } \\
\text { - Financial savings through bundled service } \\
\text { contracts }\end{array}$ \\
- Streamlined technologist education & \\
- Streamlined examination assignment and \\
resource allocation
\end{tabular}

requirement for patients prior to sedation, early morning start times are preferable for many young children to avoid prolonged times without nutrition. Space and staff must be available to ensure post-anesthesia recovery of patients [11]. In addition, for many children with neurologic conditions or developmental delay, the anesthesia MRI event is an opportunity to perform additional medical or preventative procedures while the child is under sedation, such as lumbar punctures and dental exams. An anesthesia MRI service should establish what, if any, ancillary procedures can be performed after anesthesia MRI as well as a standardized process for requesting and accommodating them.

\section{Approaches to pediatric magnetic resonance imaging process improvement}

Operating an effective MRI service requires quality assurance and improvement, which entails a collaborative team consisting of, but not limited to, radiologists, physicists, technologists, nurses and vendor specialists who have expertise in MR image acquisition, operational logistics and safety. The specific makeup of these teams depends largely on identifying appropriate stakeholders at each institution. One academic center established a "Best in Practice" program in which MR technologists were designated as advanced practice specialists trained with their MR vendor to gain further expertise in technical support and protocol optimization [12]. These advanced practice specialists served as liaisons between the MR vendor and the radiology department and helped to spearhead process improvement initiatives related to MRI.

It is also important to establish quantitative metrics related to a pediatric MRI service that can be monitored regularly to assess progress of process improvement initiatives. A number of MRIspecific key performance indicators have been identified, including mean inpatients not scanned per day, total outpatient MRI volume, percentage of exams started on or before scheduled time, outpatient anesthesia MRI backlog, mean and range of MR scan times, and percentage of exams completed on time
[13]. An important quantitative metric to consider is MR scanner utilization rate, defined as the ratio of the number of completed exams per given number of available timeslots over a defined period of time [4]. Utilization rate can be difficult to calculate, given scanner-specific variations in timeslot length depending on the scanner and day of the week, whether coordination with anesthesiology or cardiology for implanted devices is involved, exam type, patient phase of care, and scanner hours of operation.

As noted, a special consideration in pediatric MRI is balancing the benefits against potential risks of anesthetic medication exposure in MRI performed under sedation. It is important for pediatric MRI services to establish a formalized program to minimize anesthesia requirements for pediatric MRI [14]. One institution recently created a formalized non-sedated MRI program for pediatric patients that consisted of fixed 75-min timeslots, which allowed for pre-examination preparation and scanning, with appropriate support by child life specialists as needed [15]. While these appointment timeslots are longer than for similar routine examinations, the overall success rate was $82 \%$ [15], which not only reduced anesthesia exposure for children but also decreased appointment wait times and cost $[16,17]$.

Because patient factors play an outsize role in throughput, one of the controllable factors for continuous improvement is protocol standardization and optimization. Each MRI protocol involves creation of standardized planes, sequences, policies for contrast use, and technical parameters for specific MR exam types. Each radiology department should create its own set of standardized MR protocols as well as a standardized workflow for protocoling MRI exams in advance to minimize errors in image acquisition and scheduling of patients to incorrect resources (e.g., 3-T scanner, anesthesia timeslot, etc.). Ideally, MRI studies should be protocoled in advance because decisions such as scanner assignment, total image acquisition time, and need for general anesthesia depend on protocol type and length. MR protocoling should also ideally be harmonized with any payer authorization documentation in children with medical insurance to ensure that the MRI exam performed matches the study authorized by the insurance carrier. Further optimization can be achieved with recent advancements in auto-protocoling 
and increased sophistication of artificial intelligence and deep learning with regard to MRI scheduling and protocoling.

During image acquisition, a reliable, efficient communication platform for technologists and radiologists enables real-time problem-solving, image checking and examination time tracking. Radiology information systems often provide a real-time view of MR scans that are scheduled and in progress (Fig. 1) that can serve as a real-time MRI workflow dashboard. This allows MR technologists and operations managers to monitor scanner progress and conduct daily resource allocation while scheduling addon cases. Resource allocation for inpatient studies is particularly challenging; they are often time-sensitive and cannot be scheduled in advance and must conform to the clinical patient care routine. For these reasons, inpatient and emergent studies are uniquely demanding and must be prioritized based on acuity and wait time. The successful MRI service must decide how to prioritize acute patients referred from different clinical services and determine the weight of point of service, for example whether the child is based in the emergency room, inpatient floor, stepdown or intensive care unit, and its impact on queue positioning. These decisions require clear communication among technologists and radiologists, especially between shifts, and necessitate capabilities in real-time examination tracking, image checks and communication. For example, integrated instant messaging is strongly recommended. One consideration in the case of nonscheduled, emergent indications requiring prioritization, such as appendicitis, is to install MR equipment for dedicated use in the emergency department where resources allow.
Continued re-evaluation is vital to identifying and rectifying problems in any successful MRI service. There must exist a system of feedback to recognize and address quality and safety concerns. At the authors' institution, a quality improvement feedback tool is embedded into the radiology picture archiving and communication system to enable radiologists to provide feedback at the time of study interpretation. Regular quality and safety committee meetings are held with stakeholders in attendance; stakeholders include radiology operations leadership, radiologists, technologists and radiology trainees involved in the clinical MRI service.

COVID-19 challenged many MRI services with unique obstacles. During the putative first wave of COVID-19 in the United States between March and June 2020, many MRI services tackled issues regarding large-volume outpatient examination deferrals and rescheduling [18]. Responsible social distancing measures needed to be established for technologists, nurses, radiologists and patients at the MR scanners in addition to instituting protocols on use and distribution of personal protective equipment (PPE), with the majority of MR exams performed with anesthesia considered to be aerosol-generating procedures. These logistical challenges were compounded by a decrease in overall demand for MR examinations related to cancellation of nonurgent outpatient clinics and procedures [19].

Flexibility and adaptability are paramount as we prepare during the recovery phase and look ahead to subsequent waves beyond winter 2020/2021. Studies that were deferred initially must be

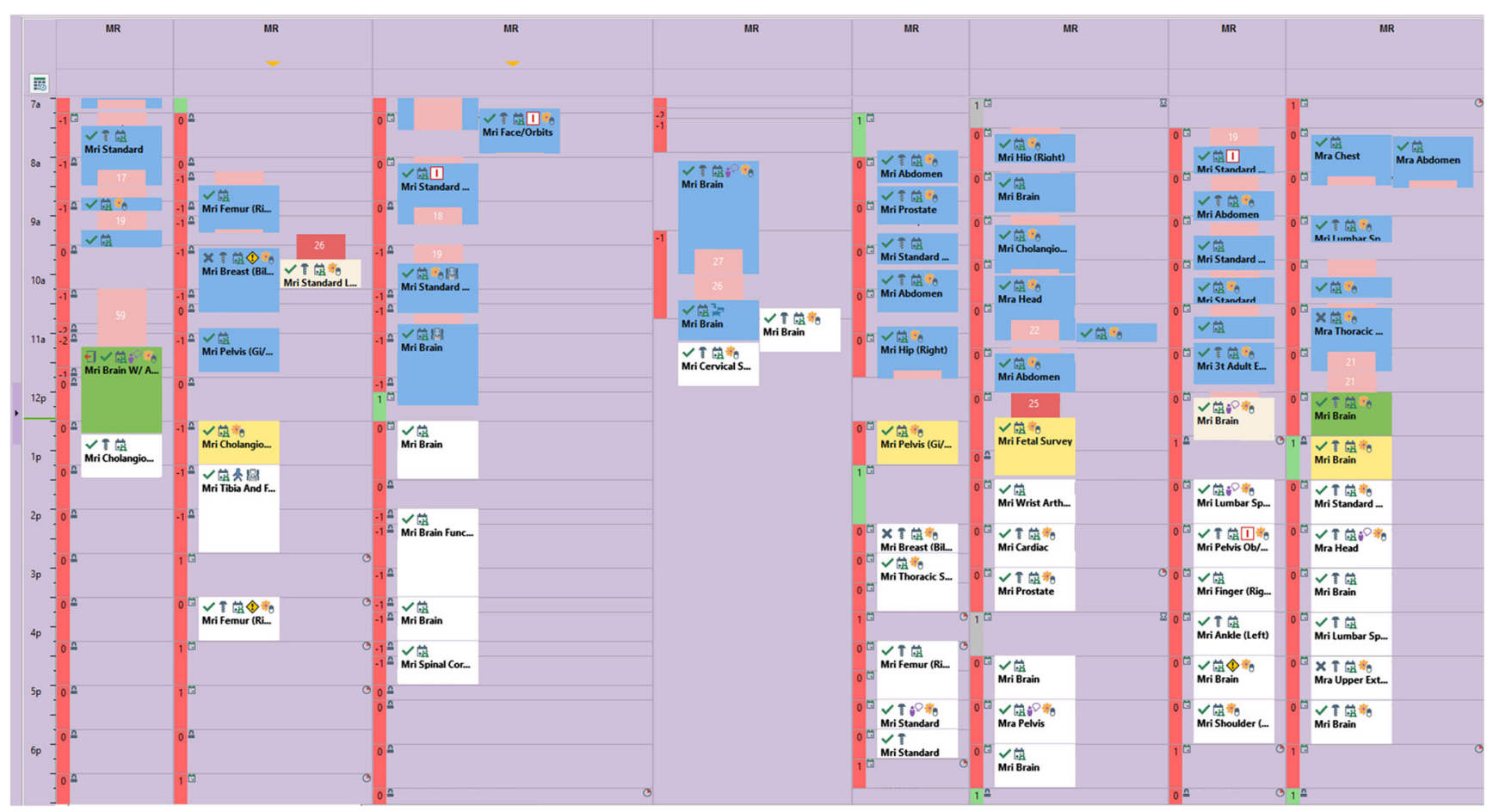

Fig. 1 An example of a real-time view of MR scans that are scheduled and in progress serving as an MRI workflow dashboard. Completed scans (blue), in-progress scans (green), in-preparation scans (yellow), scheduled scans (white) 


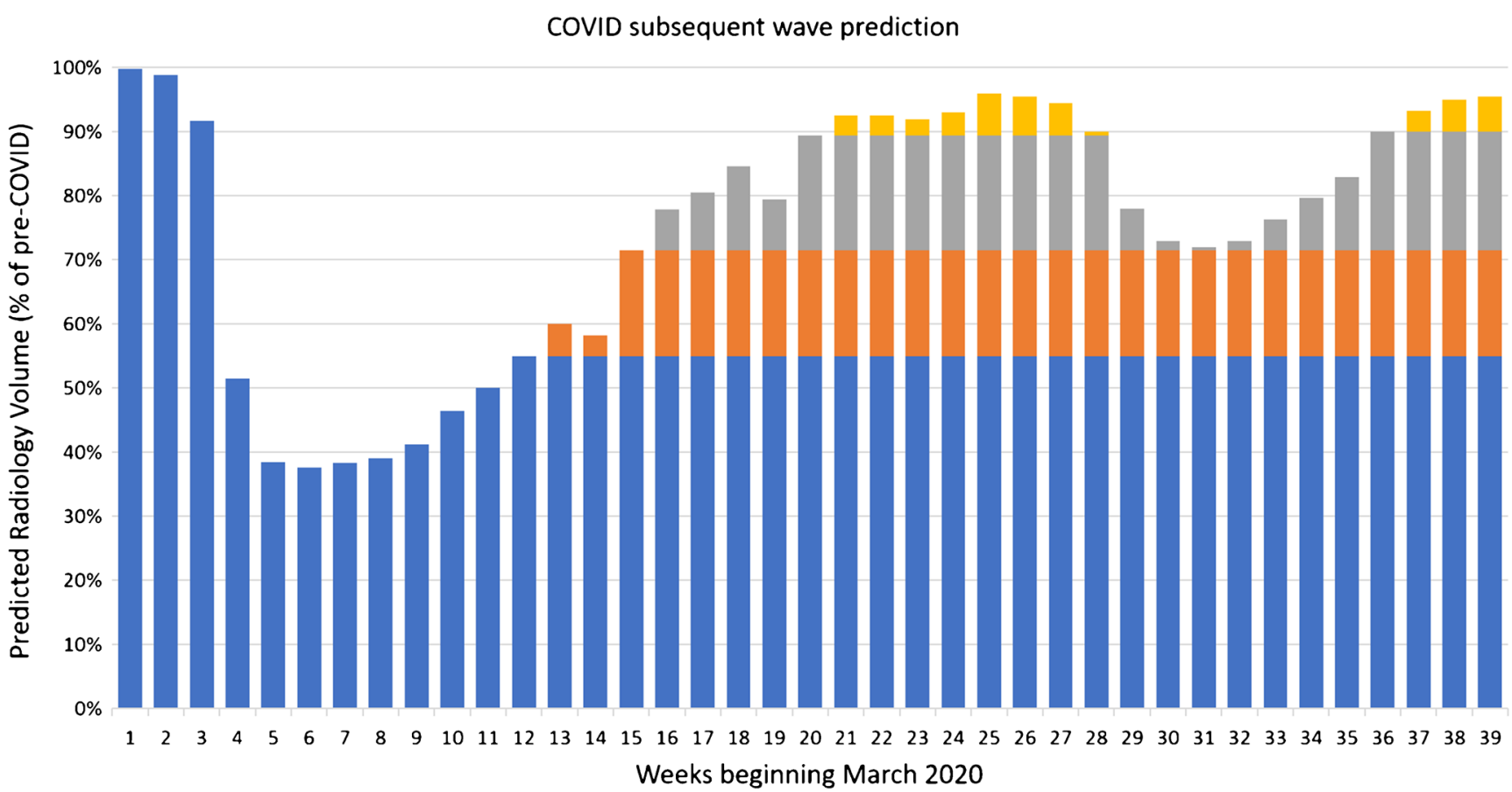

- Pandemic baseline $\square$ Reopening phase 1 measures $\square$ Reopening phase 2 measures $\square$ Reopening phase 3 measures

Fig. 2 Predictive model developed by authors' institution extrapolating the impact of local government public health reopening measures during the first wave of coronavirus disease 2019 (COVID-19) (MarchMay 2020) on anticipated radiology examination volumes as percentage of pre-COVID volumes. Each bar represents a week's worth of total exam volume. Blue bars indicate actual volume as a percentage of pre-COVID studies prior to introduction of reopening measures. Predicted volume

accommodated along with newly ordered exam requests coming from reopened clinical practices, while imaging centers continue to maintain safe practices. Radiology departments will likely need to find solutions to increase MRI capacity, through some combination of shortening MRI protocols and scan times, lengthening hours of operation, and installing new equipment. Data analytics are likely to play a crucial role in helping anticipate changes in pediatric MRI demand. The authors' institution has recently developed a predictive model extrapolating the impact of local government public health measures during the first wave of COVID19 on anticipated radiology examination volumes to subsequent waves (Fig. 2) [20].

\section{Conclusion}

A pediatric MRI service is a critically important part of a successful radiology department. A well-performing MRI service requires detailed planning that considers long-term finance, clinical operations, quality and safety, and process improvement considerations. These aspects are compounded by the unique challenges of caring for pediatric patients. In this review, we have highlighted some common issues that arise when developing a pediatric MRI service as well as a few strategies for addressing them. In the final analysis, corresponding to subsequent introduction of reopening measures is indicated by orange for phase 1, gray for phase 2 and yellow for phase 3 in order of decreased restrictions due to relaxing of COVID-19 safety protocols. Predicted decrease in volume during weeks 29-36 corresponds to potential rollback in reopening measures. Data provided by Steven Guitron, Massachusetts General Hospital Medical Analytics Group

delivering optimal patient care in an MRI service depends on a collaborative team to identify and resolve challenges and to strive for continued improvement.

Acknowledgments We thank Rick Robertson, MD, from Boston Children's Hospital and John Kirsch, PhD, from Massachusetts General Hospital/Martinos Center for Biomedical Imaging for their thoughtful input regarding radiology department-vendor relationships, and Steven Guitron and Oleg Pianykh, PhD, from the Massachusetts General Hospital Medical Analytics Group for providing the data underlying Fig. 2.

\section{Declarations}

Conflicts of interest Michael S. Gee has research funding from TakedaMillennium Pharmaceuticals.

\section{References}

1. Martí-Bonmatí L, Kormano M (1997) MR equipment acquisition strategies: low-field or high-field scanners. Eur Radiol 7:S263S268

2. Morrison LK, Siddiqi AU, Roberts WC et al (2012) A collaborative capital planning process for departments of radiology. J Am Coll Radiol 9:839-843 
3. Kozak BM, Jaimes C, Kirsch J, Gee MS (2020) MRI techniques to decrease imaging times in children. Radiographics 40:485-502

4. Thrall JH (1992) Capital planning in radiology. AJR Am J Roentgenol 159:1107-1111

5. The Royal College of Radiologists (2017) Magnetic resonance imaging (MRI) equipment, operations and planning in the NHS. The Royal College of Radiologists, London

6. Adams HG, Arora S (1994) Total quality in radiology: a guide to implementation. St. Lucie Press, Boca Raton

7. Sreedher G, Ho M-L, Smith M et al (2020) Magnetic resonance imaging quality control, quality assurance and quality improvement. Pediatr Radiol. https://doi.org/10.1007/s00247-021-05043-6

8. Jaimes C, Murcia DJ, Miguel K et al (2018) Identification of quality improvement areas in pediatric MRI from analysis of patient safety reports. Pediatr Radiol 48:66-73

9. Chandra T, Chavhan GB, Sze RW et al (2019) Practical considerations for establishing and maintaining a magnetic resonance imaging safety program in a pediatric practice. Pediatr Radiol 49:458468

10. Artunduaga M, Liu CA, Morin CE et al (2020) Safety challenges related to the use of sedation and general anesthesia in pediatric patients undergoing magnetic resonance imaging examinations. Pediatr Radiol. https://doi.org/10.1007/s00247-021-05044-5

11. Slovis TL (2011) Sedation and anesthesia issues in pediatric imaging. Pediatr Radiol 41:514-516

12. Recht M, Kim D, Lawson K, Mulholland T (2011) Best in practice: a model for improved radiologist-technologist collaboration and image optimization. J Am Coll Radiol 8:283-287
13. Recht M, Macari M, Lawson K et al (2013) Impacting key performance indicators in an academic MR imaging department through process improvement. J Am Coll Radiol 10:202-206

14. Jaimes C, Gee MS (2016) Strategies to minimize sedation in pediatric body magnetic resonance imaging. Pediatr Radiol 46:916-927

15. Jaimes C, Robson CD, Machado-Rivas F et al (2020) Success of non-sedated neuroradiological MRI in children 1 to 7 years old. AJR Am J Roentgenol. https://doi.org/10.2214/AJR.20.23654

16. Rudder BS, Easley SJ, Robinson AL et al (2019) Effects of an MRI try without program on patient access. Pediatr Radiol 49:17121717

17. Vanderby SA, Babyn PS, Carter MW et al (2010) Effect of anesthesia and sedation on pediatric MR imaging patient flow. Radiology 256:229-237

18. Naidich JJ, Boltyenkov A, Wang JJ et al (2020) Impact of the coronavirus disease 2019 (COVID-19) pandemic on imaging case volumes. J Am Coll Radiol 17:865-872

19. Rosen MP, Norbash A, Kruskal J et al (2020) Impact of coronavirus disease 2019 (COVID-19) on the practice of clinical radiology. J Am Coll Radiol 17:1096-1100

20. Guitron S, Pianykh OS, Succi MD et al (2020) COVID-19: recovery models for radiology departments. J Am Coll Radiol 17:1460 1468

Publisher's note Springer Nature remains neutral with regard to jurisdictional claims in published maps and institutional affiliations. 